\title{
The benefits and costs of biologic registers
}

\author{
David A Isenberg
}

The era of biological therapy has arrived, and we would do well to learn the lessons of the 'steroid era', in which too many years passed before the side effects of these new 'wonder drugs' were fully appreciated. Will biological agents be as good as we hope, will they have complications we have not anticipated, and will they be affordable? Pharmacovigilance is the name of the game. Furthermore, it is clear from the Vioxx ${ }^{\circledR}$ (Merck \& Co., Inc., New Jersey, NY) experience that the normal 6-12 months of pharmacovigilance carried out by pharmaceutical companies could be too short a time to make any balanced judgements.

In order to circumvent these problems, several countries-Sweden, Germany and the United Kingdom, for example-have established biological registers to determine the long-term outcome of treating patients with tumor-necrosis-factor (TNF) blockers, principally in patients with rheumatoid arthritis. The largest register established to date is the British Society for Rheumatology (BSR)'s Biologics Register (BSRBR), which will soon have been following three groups of 4,000 patients, each on etanercept, infliximab or adalimumab, and 4,000 untreated controls with rheumatoid arthritis, for at least 5 years. Individual patients' consultants are contacted twice yearly and data on patient progress and side effects are recorded. A treasure trove of biological information is already emerging, and health-economic analyses are being undertaken to determine whether, for example, the TNF blockers will, by virtue of reducing erosions and joint damage, prevent the frequent admissions to hospital for procedures such as synovectomies or joint replacements that so many patients have endured in the past.

The increasing success of the BSRBR and other European registers now brings several important questions in its wake. In particular, does it make sense to develop registers for TNFblocking drugs that are used in the treatment of less common rheumatic diseases, such as ....we need to

decide soon

whether ...

independent

pharmaco-

vigilance is

an expensive

luxury or a

necessity to

be paid for

whatever the

price

DA Isenberg is an

Advisory Board member of Nature Clinical Practice Rheumatology.

\section{Competing interests} The author declared competing interests; go to the article online for details.

www.nature.com/clinicalpractice doi:10.1038/ncprheum0166 psoriatic arthritis and ankylosing spondylitis, or even for rarer conditions, such as myositis and vasculitis? Are there sufficiently important questions to warrant separate registers for these more uncommon conditions? Furthermore, given the similarities in the form of arthritis found in many patients with psoriatic arthritis, is there a compelling case for rheumatologists (as opposed to dermatologists) to develop a separate psoriatic arthritis register? A separate register for ankylosing spondylitis seems more viable, given the major differences in disease phenotype. Another question is whether the registers should be expanded to include drugs that clearly demonstrate some benefit in the treatment of rheumatoid arthritis, such as rituximab (made by Roche) and abatacept (under development by Bristol-Myers Squibb).

Establishing and maintaining registers is expensive. Over a dozen full-time employees run the BSRBR at the Arthritis Research Centre's Epidemiology Unit in Manchester, UK. The cost of running the BSRBR of 16,000 patients with rheumatoid arthritis, for 5 years, will exceed $£ 5$ million. The arrangement established by the BSR, that the pharmaceutical companies pay for the register but cede ownership of the data to the BSR (while retaining the right to see data obtained on their own drug), is novel, and provides reassuring independent scrutiny. The current register is financially viable because four companies-Amgen, Wyeth, Schering-Plough and Abbott-contribute to it. The BSR prefers, whenever possible, for more than one company to support a register, and it is not certain that all companies would wish to contribute to other registers, raising the question of who would pay for them. Given the potential dangers of delaying the detection of possible side effects, we need to decide soon whether this kind of independent pharmacovigilance is an expensive luxury or a necessity to be paid for whatever the price. 